\title{
Epilithic diatoms (Bacillariophycae) from streams in Ramsar, Iran
}

\author{
Ali Soltanpour-Gargari ${ }^{1}$, Martin Lodenius ${ }^{1 *}$, Friedel Hinz ${ }^{2}$ \\ ${ }^{1}$ Department of Environmental Sciences, University of Helsinki, P.O.B 27, \\ FIN-00014 Helsinki, Finland \\ 2 Alfred Wegener Institute for Polar and Marine Research, Postfach 1201 61, \\ D-27515 Bremerhaven, Germany
}

\begin{abstract}
Epilithic diatoms were identified from five small streams and one canal in Ramsar, northwest Iran. A total of 155 diatom taxa belonging to 37 genera were found and only two species remained unidentified (Fragilaria sp. and Nitzschia sp.). Achnanthes, Nitzschia, Navicula, Cocconeis, Melosira, Amphora, Craticula, Diatoma, Surirella, Cymbella, Diploneis and Entomoneis were among the most abundant genera. Eighty seven taxa were recorded for the first time in Iran. Thirty two of the genera belong to the Pennales and 5 to the Centrales. Species richness was rather high ranging from 66 to 95 taxa at the six sites studied. The epilithic diatom species found in Ramsar were dominated by cosmopolitan taxa found in meso- to fairly eutrophic waters with high conductivity and high nutrient concentrations. The abundances found at all six sites were compiled in order to estimate the overall abundance of each taxon in Ramsar. This study includes EM pictures of diatoms observed in Ramsar, Iran.
\end{abstract}

Keywords: diatom, stream, Ramsar, Iran

\section{Introduction}

The diversity of freshwater diatoms is enormous (PATRICK and REIMER 1966, LANGE-Bertalot 2001, Krammer 2002). Although algae constitute a fundamental part of the aquatic food chains, only few research papers deal with the freshwater or marine diatoms of Iranian environment. Some authors have studied the seasonal distribution of phytoplankton (SoltanPour-Gargari 1973, Kimball and Kimball 1977). Hirano (1973) has studied the freshwater algae of Mesopotamia including nine sampling sites in Iran. WASYLIK (1975) and COMPÈre (1981) have studied algae in general while AlFinITO and FuMANTI (1980) reported brief systematic results from a single sampling. Diatoms were used as an indicator of pollution in a small section of the Zayandeh River in Isfahan, Iran by MogHADAM

* Corresponding author, e-mail: martin.lodenius@helsinki.fi

Copyright ${ }^{\circledR} 2011$ by Acta Botanica Croatica, the Faculty of Science, University of Zagreb. All rights reserved. 
(1976). In recent years NEJADSATTARI (2005) has studied the diatom flora of Neure Lake in East Azerbaijan, Iran. NEJADSATTARI et al. (2005) studied the composition and seasonal distribution of epiphytic algae in Anzali lagoon. ATAZADEH et al. (2007) have calculated the trophic diatom index for assessing water quality in the Gharasou River, western Iran. WASYLIKOWA and WITKOWSKI (2008) have studied fossil diatoms of Lake Zaribar in western Iran. The diatoms of the coastal area of the Caspian Sea with hundreds of large and small tributaries have received little attention although diatoms, especially the freshwater species, are the major group of algae. The aim of this study is to assess the diatom flora of some Iranian freshwater ecosystems and evaluate the relation to water quality.

\section{Material and methods}

\section{Study areas}

Epilithic diatoms were collected from one river, four streams and one canal in Ramsar in spring 2007. The location of the sampling sites was determined in such a way as to avoid direct human influence: as far away as possible from roads but still accessible. Ramsar is located in the state of Mazandaran on the coast of Caspian Sea. The city of Ramsar borders the Caspian Sea to the north, Gilan Province to the west, Qazvin Province to the south and Tonekabon city to the east (Fig. 1). We found no published or unpublished reports on water quality in this area. Due to lack of facilities and authority we were unfortunately not able to analyze the water quality in situ.

The climate is semitropical with very high temperatures and high humidity especially in summer. Geologically, this area is relatively young containing uraniferous igneous rocks from the Precambrian to the Quaternary (BOZORGNIA 1970). Ramsar is predominately a rural area and over $50 \%$ of the local population is directly or indirectly involved in farming and agricultural activities e.g. in rice fields and citrus fruit and kiwi orchards. Dumping of organic and domestic garbage along road sides is very common. In addition, Ramsar attracts a lot of tourists every year which aggravates the environmental pollution.

The city is located between the Alborz Mountains and to the west of the Caspian coastal plain. The coastal plain in Ramsar is very narrow and the city extends on the hilly terrain to the south. The bedrock of this terrain is sandstone, covered with dense forests and the annual precipitation is $1500 \mathrm{~mm}$. The weak lithology, high rainfall, high degree of weathering and also human activities such as deforestation, road construction, housing and agriculture, have increased in potential of slope instability in this area (HAFEZI MogHADDAS and GAFOORI 2007).

Description of the sampling sites (sampling date 24 March 2007):

Site 1: Sample collected from the concrete wall of a canal. Width of the canal is approximately $80 \mathrm{~cm}$. Depth is not more than $50 \mathrm{~cm}$. Fast flowing, clear water.

Site 2: Small stream near the old road to the Hotel Ramsar. Width of the stream is about $3 \mathrm{~m}$ and the depth is not more than $30 \mathrm{~cm}$. River bed is composed of pebbles and different size rocks. Water is clear.

Site 3: The same stream but about 300 meters down the road. Width is not more than $3 \mathrm{~m}$. Depth is not more than $30 \mathrm{~cm}$. At this location the water is mixed with a cooled hot spring stream. Water is clear. 


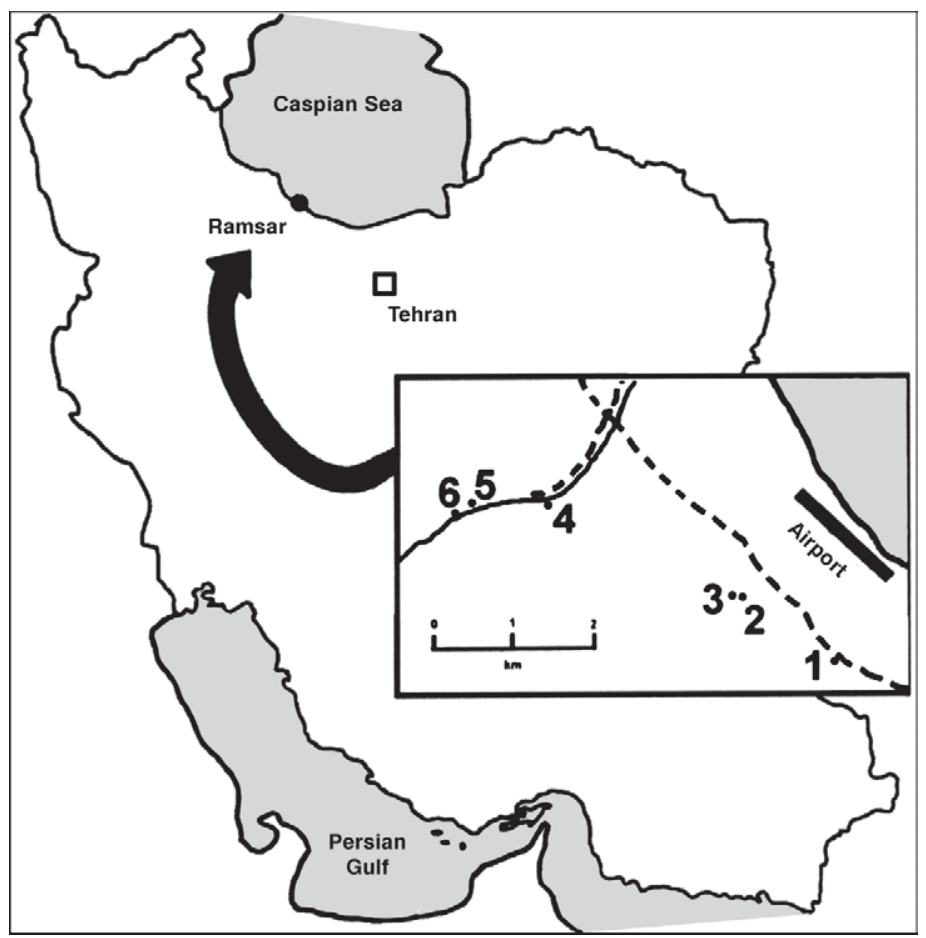

Fig. 1. The study area in Ramsar, North-West Iran.

Site 4: This site is located near the road of Javaherdeh. Due to the flood of the main river, the diatom sample was collected from the stream Gavramak 50 meters from where it joins the main river. Width is not more than $2 \mathrm{~m}$. River bed is composed of fine pebbles, small stones and large boulders. Depth is not more than $20 \mathrm{~cm}$. Water clear.

Site 5: This site is also in the Javaherdeh road. The water runs down a hill. Stones are covered with moss and green algae. Width is about 2 meters at sampling site. Depth is not more than $10 \mathrm{~cm}$. Water is clear.

Site 6: Sample was taken from a small stream, which runs through the road. Width is not more than $2 \mathrm{~m}$. Depth at sampling site is about $20 \mathrm{~cm}$. River bed is composed of medium size pebbles and small stones. Water is clear.

\section{Diatom sampling and identification}

At each sampling location diatoms were collected by removing submerged stones and by scraping their surface using a plastic spatula and a plastic bottle. The samples were diluted with stream water and later preserved with $5 \%$ formaldehyde.

In the laboratory of the Department of Environmental Sciences of Helsinki University sub samples of diatoms were digested with sulphuric / nitric acid (4/1) in order to eliminate organic matter. Samples were then washed by decanting using distilled water.

For permanent diatom slides the cleaned and rinsed diatom samples were mounted on glass slides using NAPHRAX® $(i=1.74)$. A Zeiss microscope with full immersion objec- 
tives was used for diatom identification at $1000 \mathrm{x}$ magnification. OMIT (no ligth microscope pictures are used). The electron microscope photos were obtained in Alfred Wegener Institute (Germany) with a Scanning Electron Microscope FE1 Quanta 200 FEG. In order to determine the relative frequency of commonly occurring taxa, 3 slides were prepared for each sampling site, and in each slide 300 valves were counted along randomly selected transects. To achieve maximum taxa diversity, two slides from each sampling sites have been examined in detail.

The following references were used for diatom identification: KRAMMER (1992; 1997a, b, 2002); Krammer and LANGe-Bertalot (1986; 1988; 1991a, b); LANGe-Bertalot (1993, 2001); LANGE-Bertalot and Metzelin (1996) and Reichardt (1999).

Diatoms were scored on a semi quantitative scale according to VESELÁ and JOHANSEN (2009): rare (one or two frustules per sample), uncommon (less than $5 \%$ of cells), common $(5-25 \%)$, frequent $(25-50 \%)$, and dominant more than $50 \%$ of cells.

\section{Results}

A total of 153 diatom taxa were identified from the Ramsar samples. This study originally covered a total of 153 identified species from the Ramsar samples with some presented in Plates 1-4, Figs. 2-33. Eighty seven of the taxa observed in the present study have not been previously reported in Iran. Two species (Fragilaria sp. and Nitzschia sp.) remained unidentified.

\section{Taxonomic diversity}

Thirty two of the genera belong to the Pennales and 5 to the Centrales. Three of the Centrals were not reported in Iran before: Ellerbeckia arenaria f. arenaria, Orthoseira roeseana and Pleurosira laevis f. polymorpha. Melosira varians was present almost at all sites with $7 \%$ of the overall population (Tab. 1). Two species of Cyclotella (C. comensis and $C$. meneghiniana) were also observed. Nitzschia with 24 species showed the highest species diversity in the Pennales, followed by Navicula with 20 species. The list of all taxa (Tab. 2) including a semi-quantitative scale of abundance is in alphabetical order and shows diatom species found in Ramsar. Bacillaria, Craticula, Entomoneis, Eunotia, Frustulia, Hantzschia, Haslea, Mastogloia, Meridion, Pleurosigma, Rhoicosphenia, Stenopterobia and Synedra were represented by only one species each.

The abundances found at all six sites were compiled in order to estimate the overall abundance of each taxon in Ramsar. Achnanthes, Nitzschia, Navicula, Cocconeis, Melosira, Amphora, Craticula, Diatoma, Surirella, Cymbella, Diploneis and Entomoneis were among the most abundant genera (Tab. 3). Twelve species were particularly abundant: Achnanthes minutissima var. jackii, Navicula phylleptosoma, Melosira varians, Cocconeis placentula var. euglypta, Nitzschia palea, Nitzschia constricta, Cocconeis pediculus, Amphora pediculus, Navicula salinarum var. salinarum, Craticula buderi, Nitzschia inconspicua and Navicula cryptocephala. All sites contained diatom species characteristic of more alkaline and nutrient rich waters, with the exception of Frustulia rhomboides var. crassinervia (acidobiontic) which was rare, Fragilaria tenera (acidophilous) as a rare taxon only at site 3 and Stenopterobia curvula (acidophilous) as a rare taxon at sites 2 and 3. 


\section{EPILITHIC DIATOMS FROM RAMSAR, IRAN}
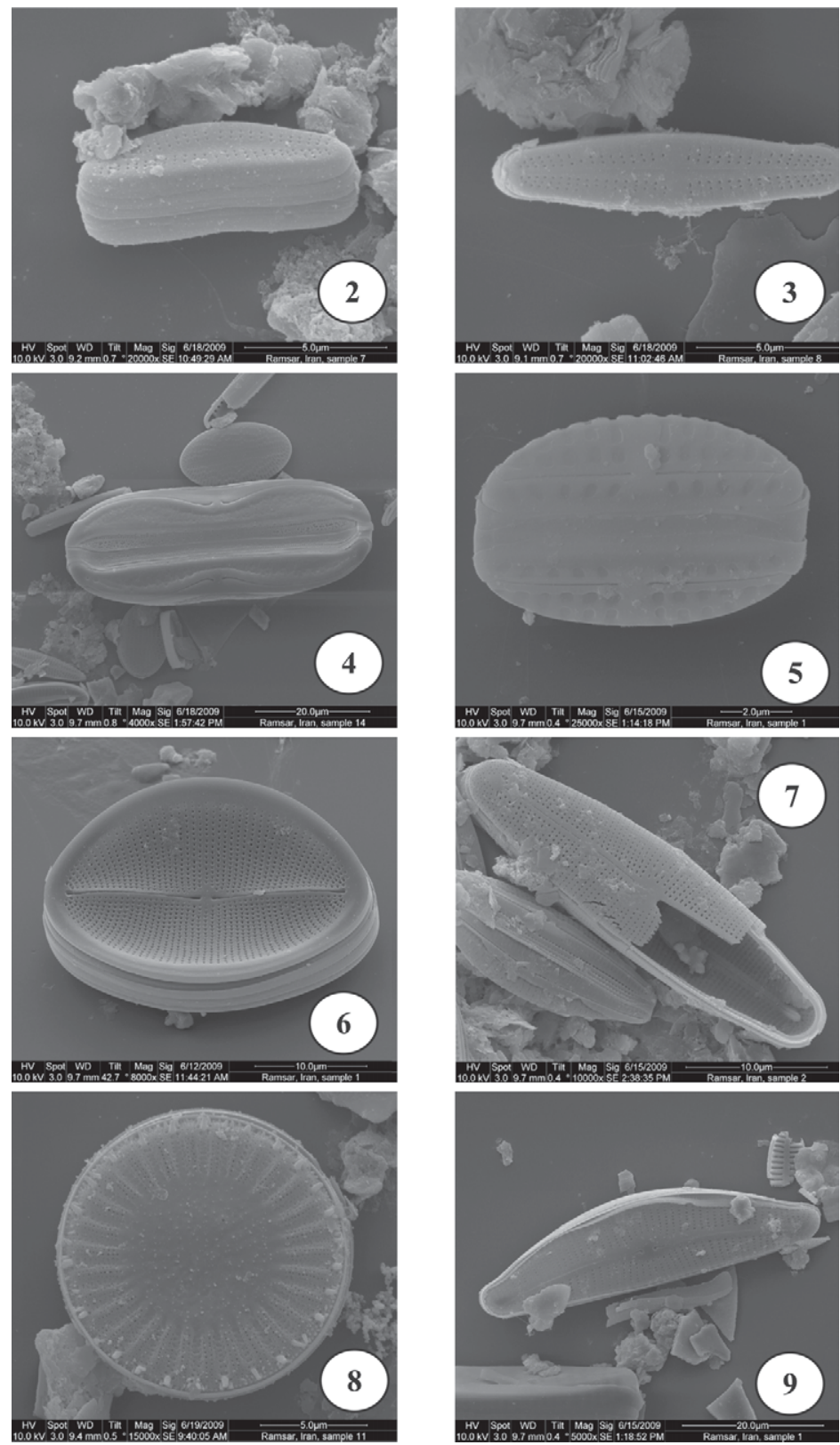

Plate 1. Figs. 2-3. Achnanthes minutissima var. jackii (Rabenhorst) Lange-Bertalot 1980. Length $15 \mu \mathrm{m}$, breadth $3.5 \mu \mathrm{m}$, striae $26 / 10 \mu \mathrm{m}$; Fig. 4 . Amphora commutata Grunow 1880. Length $52 \mu \mathrm{m}$, breadth $12 \mu \mathrm{m}$, striae 13/10 $\mathrm{m}$. Fig. 5. Amphora pediculus (Kützing) Grunow1880. Length $10 \mu \mathrm{m}$, breadth $5 \mu \mathrm{m}$, striae 18/ $10 \mu \mathrm{m}$; Fig. 6. Cocconeis pediculus Ehrenberg 1838. Length $27 \mu \mathrm{m}$, breadth $23 \mu \mathrm{m}$, striae $22 / 10 \mu \mathrm{m}$; Fig. 7. Craticula buderi (Hustedt) Lange-Bartalot 2000. Length $31 \mu \mathrm{m}$, breadth $8 \mu \mathrm{m}$, striae 21/10 $\mu \mathrm{m}$; Fig. 8. Cyclotella meneghiniana Kützing 1844. Diameter $12 \mu \mathrm{m}$; Fig. 9. Cymbella turgiduliformis Krammer 2002. Length $47 \mu \mathrm{m}$, breadth $14 \mu \mathrm{m}$, striae $13 / 10 \mu \mathrm{m}$ 

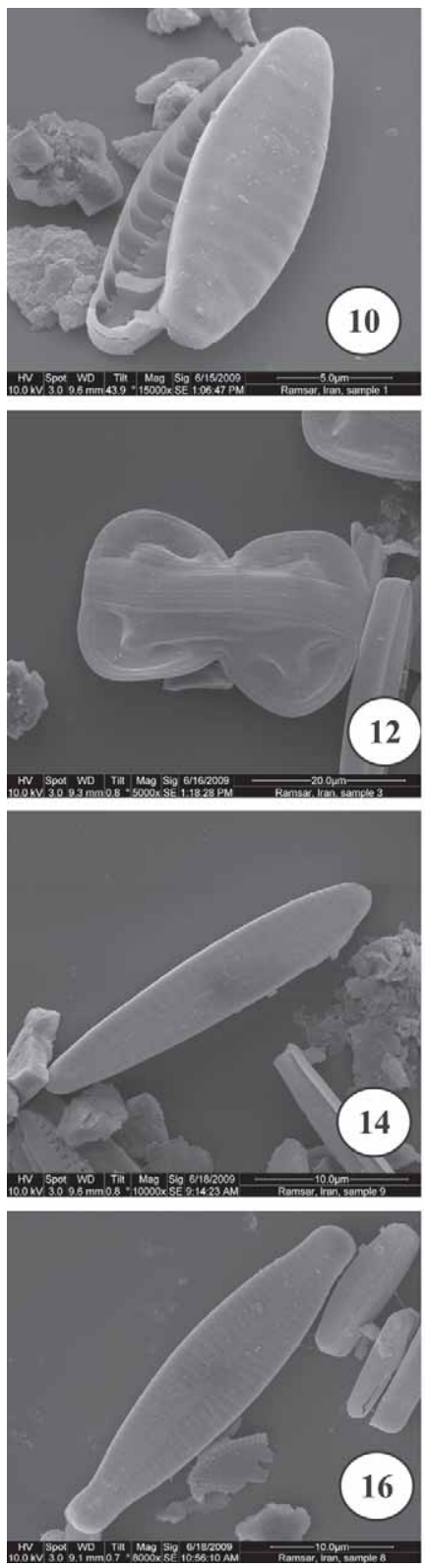
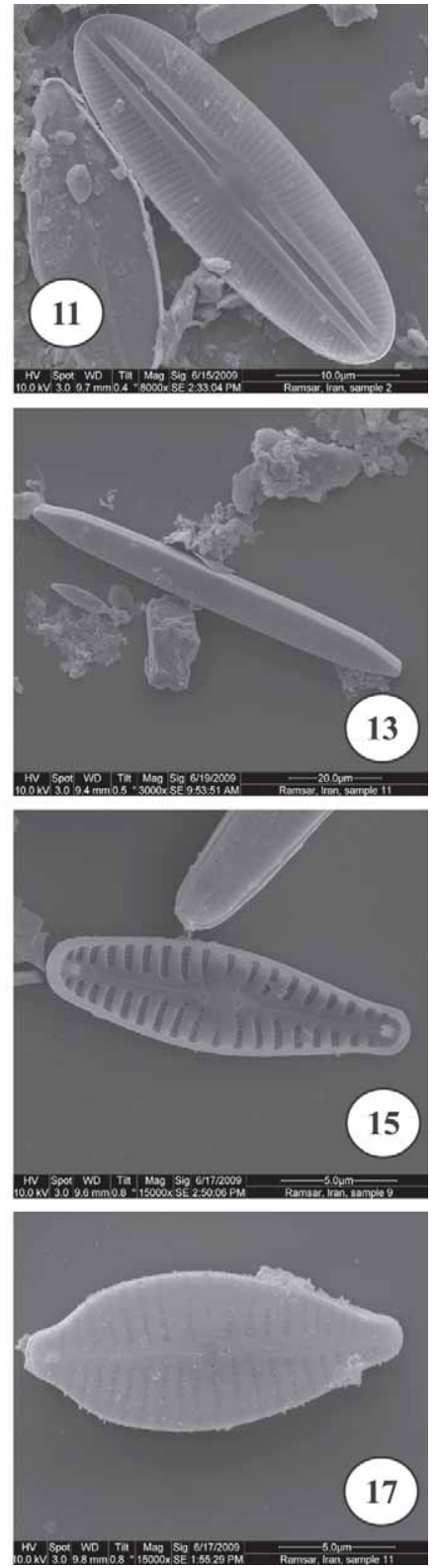

Plate 2 Fig. 10. Diatoma vulgaris Morphotype vulgaris Bory 1824. Length $15 \mu \mathrm{m}$, breadth $7 \mu \mathrm{m}$; Fig. 11. Diploneis oblongella (Naegeli) Cleve-Euler 1922. Length $36 \mu \mathrm{m}$, breadth $9 \mu \mathrm{m}$, striae 19/10 $\mu \mathrm{m}$; Fig. 12. Entomoneis paludosa var. subsalina (Cleve) Krammer 1986. Length $60 \mu \mathrm{m}$, breadth $27 \mu \mathrm{m}$, striae 20/10 $\mu \mathrm{m}$; Fig. 13. Fragilaria ulna var. ulna (Nitzsch) Lange-Bertalot 1980. Length $83 \mu \mathrm{m}$, breadth $7 \mu \mathrm{m}$, striae 9/10 $\mu \mathrm{m}$; Fig. 14. Gomphonema angustatum (Kützing) Rabenhorst 1864. Length $25 \mu \mathrm{m}$, breadth $5 \mu \mathrm{m}$, striae $11 / 10 \mu \mathrm{m}$; Fig. 15. Gomphonema olivaceum var. olivaceum (Hornemann) Brebisson 1838. Length $30 \mu \mathrm{m}$, breadth $7 \mu \mathrm{m}$, striae 12/10 $\mu \mathrm{m}$; Figs. 16-17. Gomphonema parvulum Kützing 1849. Length $35 \mu \mathrm{m}$, breadth $9 \mu \mathrm{m}$, striae $13 / 10 \mu \mathrm{m}$. 

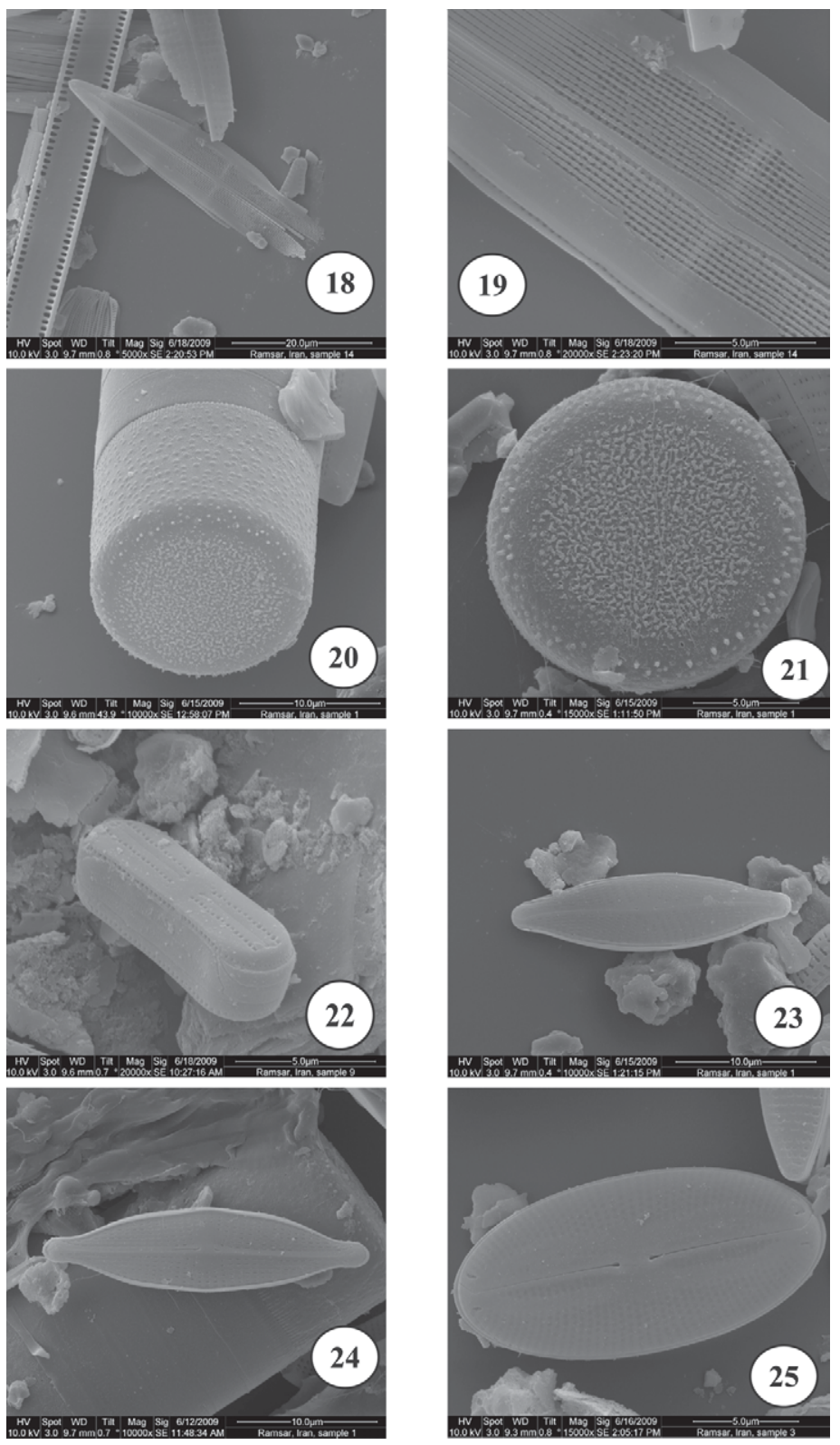

Plate 3 Figs. 18-19. Haslea spicula (Hickie) Lange-Bertalot 1997. Length $52 \mu \mathrm{m}$, breadth $10 \mu \mathrm{m}$, striae 32/10 $\mu \mathrm{m}$; Figs. 20-21. Melosira varians Agardh 1827. Diameter/15 $\mu \mathrm{m}$ Mantelhohe $11 / 10 \mu \mathrm{m}$; Fig. 22. Navicula contenta Grunow in Van Heurck 1884-1887. Length $9 \mu \mathrm{m}$, breadth $2.5 \mu \mathrm{m}$, striae $-/ 10 \mu \mathrm{m}$; Fig. 23. Navicula cryptocephala Kützing 1844. Length 20 $\mu \mathrm{m}$, breadth $6 \mu \mathrm{m}$, striae 15/10 $\mu \mathrm{m}$; Fig. 24. Navicula phylleptosoma Lange-Bertalot 1999. Length $24 \mu \mathrm{m}$, breadth $6 \mu \mathrm{m}$, striae 17/10 $\mu \mathrm{m}$; Fig. 25. Navicula pygmaea Kützing 1849. Length $20 \mu \mathrm{m}$, breadth $9 \mu \mathrm{m}$, striae $-/ 10 \mu \mathrm{m}$. 

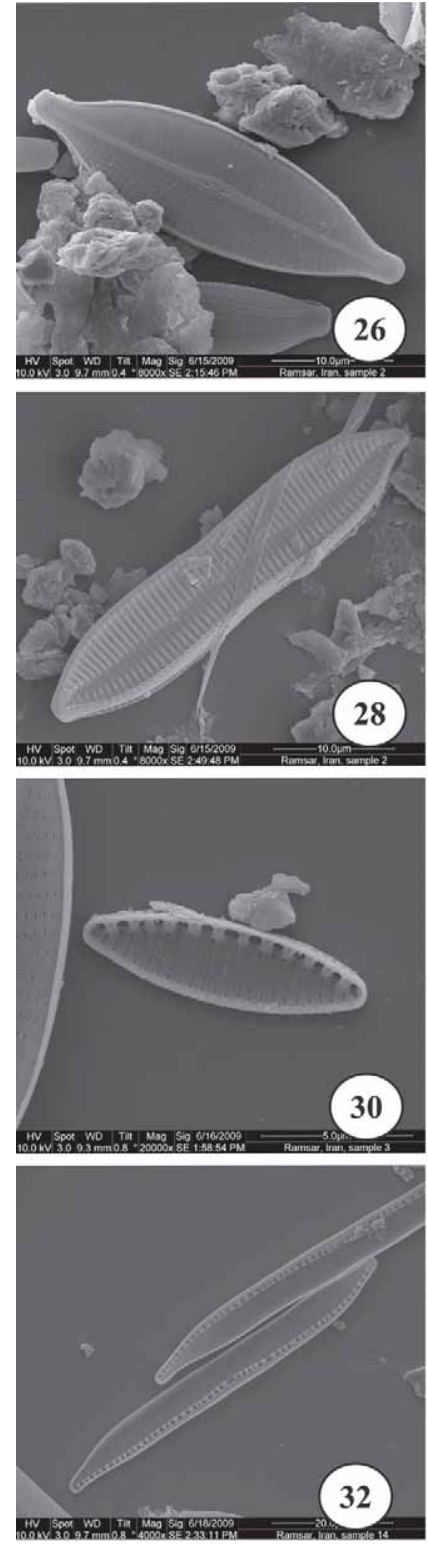
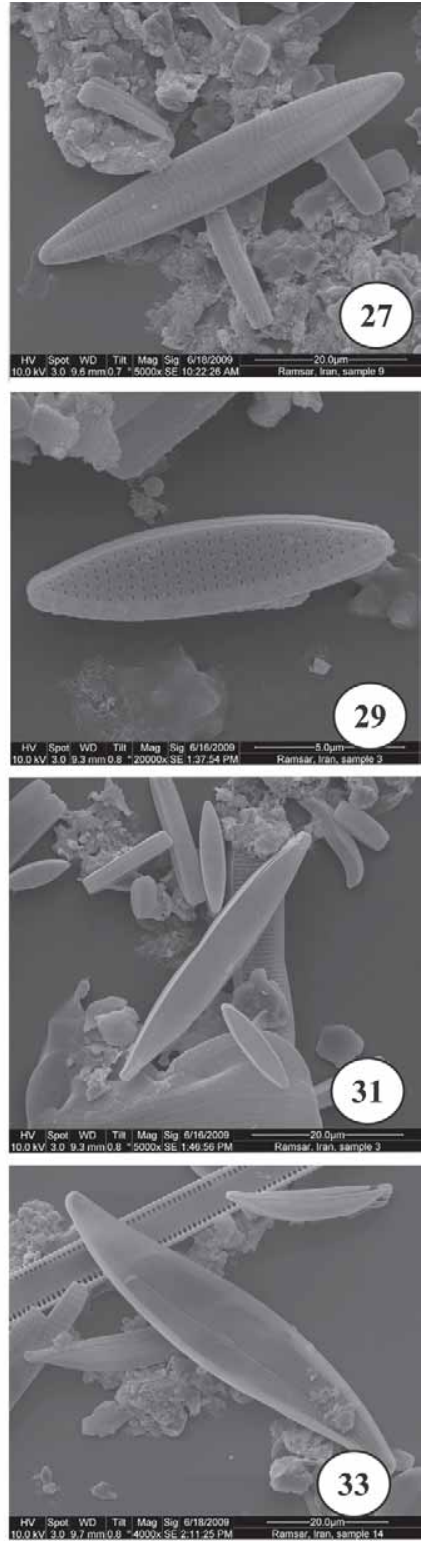

Plate 4 Fig. 26. Navicula salinarum var. salinarum Grunow 1880. Length $33 \mu \mathrm{m}$, breadth $11 \mu \mathrm{m}$, striae 17/10 $\mu \mathrm{m}$; Fig. 27. Navicula tripunctata (O.F.Müller) Bory 1822. Length $67 \mu \mathrm{m}$, breadth $9 \mu \mathrm{m}$, striae 11/10 $\mu \mathrm{m}$; Fig. 28. Nitzschia constricta (Kützing) Ralf in Pritchard 1861. Length $30 \mu \mathrm{m}$, breadth $6 \mu \mathrm{m}$, striae $18 / 10 \mu \mathrm{m}$; Fig. 29. Nitzschia frustulum var. frustulum (Kützing) Gronow 1880. Length $13 \mu \mathrm{m}$, breadth $3.2 \mu \mathrm{m}$, striae 18/10 $\mu \mathrm{m}$ Fibulaea 11/10 $\mu \mathrm{m}$; Fig. 30. Nitzschia inconspicua Grunow 1862. Length $10 \mu \mathrm{m}$, breadth $2.5 \mu \mathrm{m}$, striae $28 / 10 \mu \mathrm{m}$ fibulaea $12 / 10$ $\mu \mathrm{m}$; Fig. 31. Nitzschia palea (Kützing) W. Smith 1856. Length $43 \mu \mathrm{m}$, breadth $4.5 \mu \mathrm{m}$, Fibulae $15 / 10 \mu \mathrm{m}$; Fig. 32. Nitzschia scalpelliformis (Grunow) Grunow 1880. Length $60 \mu \mathrm{m}$, breadth $5 \mu \mathrm{m}$, striae 36/ $10 \mu \mathrm{m}$ fibulaea 12/10 $\mu \mathrm{m}$; Fig. 33. Pleurosigma salinarum Grunow 1880. Length $70 \mu \mathrm{m}$, breadth $16 \mu \mathrm{m}$, striae $-/ 10 \mu \mathrm{m}$. 
Tab. 1: Mean percentages of more commonly occurring diatom taxa at each sampling site.

\begin{tabular}{lcccccc}
\hline \multirow{2}{*}{ Species } & \multicolumn{7}{c}{ sampling sites (mean \%) } \\
\cline { 2 - 7 } & 1 & 2 & 3 & 4 & 5 & 6 \\
\hline Achnanthes minutissima var. jackii & $<1$ & - & - & 39 & 51 & 32 \\
Amphora pediculus & 2.4 & 1 & $<1$ & 7.6 & $<1$ & 11 \\
Cocconeis pediculus & 10.4 & $<1$ & $<1$ & 16.1 & - & 1.2 \\
C. placentula var. euglypta & 4.7 & $<1$ & 1.7 & 12 & 5.7 & 11 \\
Craticula buderi & $<1$ & - & - & - & 19.3 & - \\
Melosira varians & 32.3 & 2 & 4.5 & 1 & $<1$ & 1.7 \\
Navicula cryptocephala & 2 & 1 & 6.2 & 3 & $<1$ & 6.2 \\
N. phylleptosoma & - & 40 & 1 & - & - & - \\
N. salinarum var. salinarum & $<1$ & 20.3 & $<1$ & - & - & $<1$ \\
Nitzschia constricta & - & 1.6 & 27.3 & - & - & - \\
N. inconspicua & $<1$ & 1.4 & 16.7 & $<1$ & $<1$ & $<1$ \\
N. palea & $<1$ & - & $<1$ & $<1$ & 14 & 17 \\
\hline
\end{tabular}

\section{Discussion and conclusions}

As the present study carried out in Ramsar area is the first to observe the taxonomy of epilithic diatoms, it is hard to formulate any comparison with the diversity found in other regions. However, the general species composition of the diatoms recorded in Ramsar is mainly in agreement with that reported for the northern hemisphere. The majority of identified taxa are classified as alpine species (KRAMMER and LANGE-BERTALOT 1986, 1996). The epilithic diatom species found in Ramsar were dominated by cosmopolitan taxa found in waters with meso- to fairly eutrophic water with high conductivity and high nutrient concentrations. All the abundant species from table 3 have been characterized by VAN DAM et al. (1994) with exception of Craticula buderi and Navicula phylleptosoma, which were characterized by LANGE-BERTALOT (2001). Based on the taxa observed, the sampled canal and streams were established as being meso- to eutrophic waters with circumneutral to alkaline $\mathrm{pH}$, elevated or periodically elevated with a concentration of organically bound nitrogen and oxygen level from moderate (above 50\% saturation) to fairly high (above $75 \%$ saturation). According to FORE and GRAFE (2002), increase of alcaliphilic diatoms is due to the increasing intensity of agriculture and livestock grazing. Evaporation of the irrigation water in the agricultural fields can leave salt or alkaline residue that is washed into the rivers. In addition, fertilization and erosion can increase nutrient contents and alkalinity (KHAN and ALI 2003). Most frequent taxa occurring in all sites were Achnanthes minutissima var. minutissima, Amphora pediculus, Cocconeis pediculus, Navicula cryptocephala, Navicula tripunctata and Rhoicosphenia abbreviate (Tab. 2). These taxa are common species of the benthic diatom community and have been reported from other parts of Iran (Nejadsattari 2005, Compère 1981, Moghadam 1976, Wasylik 1975).

There were clear differences between different sites. Species diversity was rather high ranging from 66 to 95 taxa. The highest number of species was recorded at site 3 (95 taxa) 
Tab. 2. List of diatom species from Ramsar and their semi-quantitavie sacle of abundance: $r=$ rare,$u=$ uncommon, $c=$ common, $f=$ frequent, $d=$ dominant; $\mathrm{pH}$ iv = Van Dam's pH indication values: $1=$ acidobiontic, $2=$ acidophilous, $3=$ circumneutral, $4=$ alkaliphilous, $5=$ alkalibiontic, $6=$ indifferent; * $=$ reported, ${ }^{* *}=$ reported for the first time in Iran

\begin{tabular}{|c|c|c|c|c|c|c|c|c|c|c|c|c|c|}
\hline Taxon & Site 1 & Site 2 & Site 3 & Site 4 & Site 5 & Site 6 & $\mathrm{pH}$ iv & $\begin{array}{l}\text { this } \\
\text { study }\end{array}$ & $\begin{array}{c}\text { Nejad- } \\
\text { sattari } \\
2005 \\
\end{array}$ & $\begin{array}{c}\text { Com- } \\
\text { pére } \\
1981\end{array}$ & $\begin{array}{c}\text { Mogah- } \\
\text { dam } \\
1976 \\
\end{array}$ & $\begin{array}{c}\text { Wasylik } \\
1975\end{array}$ & $\begin{array}{c}\text { Hirano } \\
1973\end{array}$ \\
\hline
\end{tabular}

Achnantes brevipes var. intermedia (Kützing) Cleve

A. coaretata (Brébisson) Grunow

A. inflata (Kützing) Grunow

A. lanceolata ssp. biporoma (Hohn et Hellermann) Lange-Bertalot

A. lanceolata ssp. frequentissima var. frequentissima Lange-Bertalot

A. lanceolata ssp. lanceolata var. boyei (Oestrup) Lange-Bertalot

A. lanceolata ssp. lanceolata var. haynaldii (Schaarschmidt) Cleve

A. lanceolata ssp. lanceolata var. lanceolata (Brébisson) Grunow

A. minutissima var. Jackii (Rabenhorst) Lange-Bertalot

A. parvula Kützing

A. ploenensis var. gessneri (Hustedt) Lange-Bertalot

Amphora coffeaeformis var. coffeaeformis (Agardh) Kützing

A. commutata Grunow

$\begin{array}{ccccccccc}\mathrm{r} & \mathrm{r} & \mathrm{r} & \mathrm{r} & \mathrm{r} & \mathrm{r} & - & * * \\ - & - & \mathrm{r} & \mathrm{r} & \mathrm{r} & \mathrm{r} & 3 & * * \\ - & - & - & \mathrm{r} & - & \mathrm{r} & - & * * \\ \mathrm{r} & \mathrm{r} & - & - & \mathrm{r} & \mathrm{r} & 3 & * * \\ - & \mathrm{r} & - & \mathrm{r} & \mathrm{r} & \mathrm{r} & 4 & * *\end{array}$

r
-
-




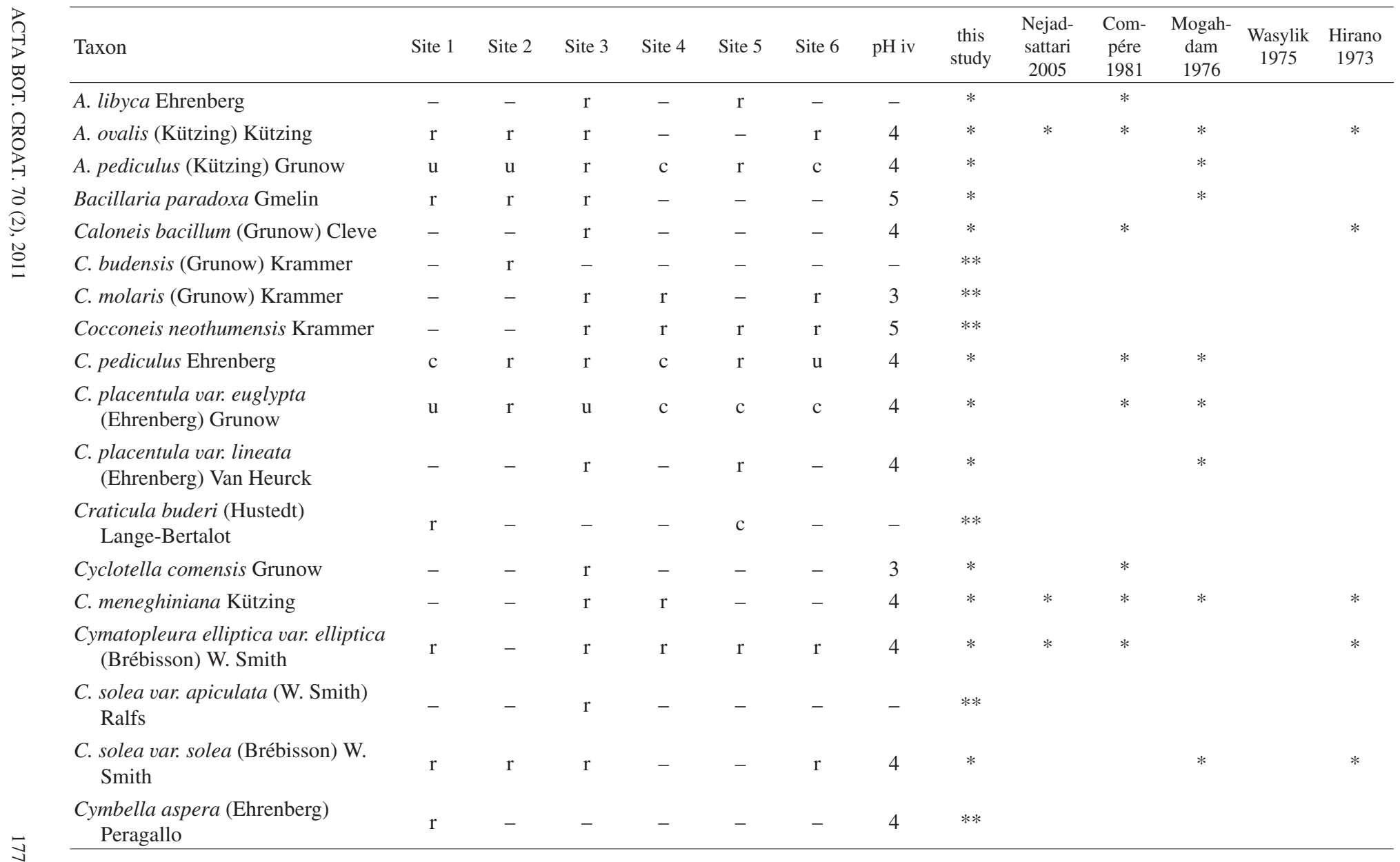




\begin{tabular}{|c|c|c|c|c|c|c|c|c|c|c|c|c|c|}
\hline Taxon & Site 1 & Site 2 & Site 3 & Site 4 & Site 5 & Site 6 & $\mathrm{pH}$ iv & $\begin{array}{l}\text { this } \\
\text { study }\end{array}$ & $\begin{array}{c}\text { Nejad- } \\
\text { sattari } \\
2005\end{array}$ & $\begin{array}{c}\text { Com- } \\
\text { pére } \\
1981 \\
\end{array}$ & $\begin{array}{c}\text { Mogah- } \\
\text { dam } \\
1976 \\
\end{array}$ & $\begin{array}{c}\text { Wasylik } \\
1975\end{array}$ & $\begin{array}{c}\text { Hirano } \\
1973\end{array}$ \\
\hline C. helvetica Kützing & - & - & - & $\mathrm{r}$ & - & $\mathrm{r}$ & 4 & $*$ & & $*$ & & & $*$ \\
\hline C. minuta Hilse & $\mathrm{r}$ & $\mathrm{r}$ & $\mathrm{r}$ & $\mathrm{r}$ & $\mathrm{u}$ & $\mathrm{u}$ & 3 & $* *$ & & & & & \\
\hline $\begin{array}{l}\text { C. naviculiformis (Auerswald) } \\
\text { Cleve }\end{array}$ & - & $\mathrm{r}$ & $\mathrm{r}$ & $\mathrm{r}$ & - & $\mathrm{r}$ & - & $*$ & & & $*$ & & \\
\hline C. prostrata (Berkeley) Cleve & $\mathrm{r}$ & $\mathrm{r}$ & - & $\mathrm{r}$ & - & $\mathrm{r}$ & 4 & $*$ & & & $*$ & & \\
\hline C. simonsenii Krammer & $\mathrm{r}$ & - & $\mathrm{r}$ & - & - & - & - & $* *$ & & & & & \\
\hline C. sinuata Gregory & $\mathrm{r}$ & $\mathrm{r}$ & $\mathrm{r}$ & $\mathrm{r}$ & $\mathrm{r}$ & - & 3 & $*$ & & $*$ & $*$ & & \\
\hline C. tumida (Brébisson) Van Heurck & $\mathrm{r}$ & - & $\mathrm{r}$ & $\mathrm{r}$ & $\mathrm{r}$ & - & 4 & * & & & $*$ & & \\
\hline C. turgiduliformis Krammer & $\mathrm{r}$ & $\mathrm{r}$ & $\mathrm{r}$ & $\mathrm{c}$ & $\mathrm{c}$ & $\mathrm{r}$ & 4 & $* *$ & & & & & \\
\hline $\begin{array}{l}\text { Denticula kuetzingii var. kuetzingii } \\
\text { Grunow }\end{array}$ & - & - & - & - & $\mathrm{r}$ & - & 4 & $* *$ & & & & & \\
\hline D. tenuis Kützing & - & $\mathrm{r}$ & - & - & $\mathrm{r}$ & $\mathrm{r}$ & 4 & $* *$ & & & & & \\
\hline $\begin{array}{l}\text { Diatoma mesodon (Ehrenberg) } \\
\text { Kützing }\end{array}$ & - & - & - & $\mathrm{r}$ & - & $\mathrm{r}$ & 3 & $* *$ & & & & & \\
\hline D. moniliformis Kützing & $\mathrm{u}$ & $\mathrm{r}$ & - & $\mathrm{u}$ & $\mathrm{r}$ & $\mathrm{r}$ & - & $* *$ & & & & & \\
\hline $\begin{array}{l}\text { D. vulgaris morphotype constricta } \\
\text { Grunow }\end{array}$ & $\mathrm{r}$ & $\mathrm{r}$ & - & - & - & $\mathrm{r}$ & 5 & $* *$ & & & & & \\
\hline $\begin{array}{l}\text { D. vulgaris morphotype vulgaris } \\
\text { Bory }\end{array}$ & $\mathrm{c}$ & $\mathrm{r}$ & $\mathrm{r}$ & $\mathrm{r}$ & - & $\mathrm{r}$ & - & $*$ & $*$ & $*$ & $*$ & & \\
\hline Diploneis marginestriata Hustedt & $\mathrm{r}$ & $\mathrm{r}$ & $\mathrm{r}$ & - & $\mathrm{r}$ & - & - & $* *$ & & & & & \\
\hline D. oblongella (Naegeli) Cleve-Euler & $\mathrm{r}$ & $\mathrm{c}$ & $\mathrm{r}$ & $\mathrm{r}$ & $\mathrm{r}$ & $\mathrm{r}$ & 4 & $*$ & & $*$ & & & \\
\hline D. parma Cleve & - & $\mathrm{r}$ & - & - & - & - & - & $* *$ & & & & & \\
\hline $\begin{array}{l}\text { D. smithii var. dilatata (Peragallo) } \\
\text { Terry }\end{array}$ & - & $r$ & $\mathrm{r}$ & - & - & - & 5 & $* *$ & & & & & \\
\hline
\end{tabular}




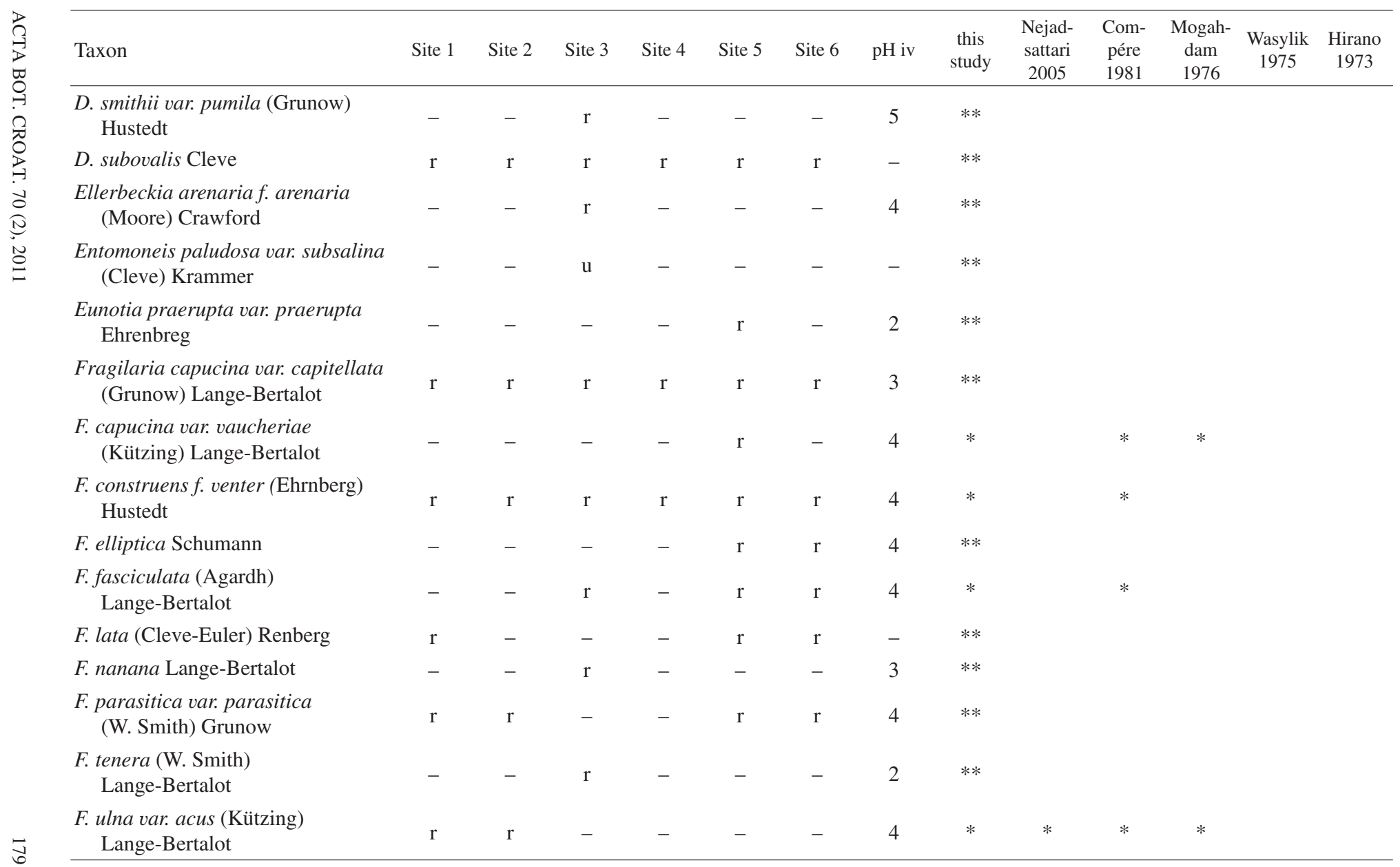




\begin{tabular}{|c|c|c|c|c|c|c|c|c|c|c|c|c|c|}
\hline Taxon & Site 1 & Site 2 & Site 3 & Site 4 & Site 5 & Site 6 & $\mathrm{pH}$ iv & $\begin{array}{l}\text { this } \\
\text { study }\end{array}$ & $\begin{array}{c}\text { Nejad- } \\
\text { sattari } \\
2005\end{array}$ & $\begin{array}{c}\text { Com- } \\
\text { pére } \\
1981\end{array}$ & $\begin{array}{c}\text { Mogah- } \\
\text { dam } \\
1976\end{array}$ & $\begin{array}{l}\text { Wasylik } \\
1975\end{array}$ & $\begin{array}{c}\text { Hirano } \\
1973\end{array}$ \\
\hline $\begin{array}{l}\text { F. ulna var. ulna (Nitzsch) } \\
\text { Lange-Bertalot }\end{array}$ & $\mathrm{r}$ & $\mathrm{r}$ & $\mathrm{r}$ & $\mathrm{r}$ & $\mathrm{r}$ & - & 4 & $*$ & $*$ & $*$ & $*$ & & $*$ \\
\hline F. ungeriana Grunow & - & - & - & - & - & $\mathrm{r}$ & - & $*$ & & $*$ & & & \\
\hline $\begin{array}{l}\text { Frustulia rhomboides var. } \\
\quad \text { crassinervia (Brébisson) Ross }\end{array}$ & $\mathrm{r}$ & - & $\mathrm{r}$ & $\mathrm{r}$ & $\mathrm{r}$ & $\mathrm{r}$ & 1 & $* *$ & & & & & \\
\hline $\begin{array}{l}\text { Geissleria ignota }(\text { Krasske) } \\
\quad \text { Lange-Bertalot et Metzeltin }\end{array}$ & - & - & - & - & $\mathrm{r}$ & - & - & $* *$ & & & & & \\
\hline $\begin{array}{l}\text { G. moseri Metzeltin, Witkowski et } \\
\text { Lange-Bertalot }\end{array}$ & - & $\mathrm{u}$ & - & - & - & - & - & $* *$ & & & & & \\
\hline $\begin{array}{l}\text { G. schoenfeldii (Hustedt) } \\
\text { Lange-Bertalot et Metzeltin }\end{array}$ & - & $\mathrm{r}$ & - & - & - & - & - & $* *$ & & & & & \\
\hline $\begin{array}{l}\text { Gomphonema affine var. affine } \\
\text { Kützing }\end{array}$ & - & - & $\mathrm{r}$ & - & - & - & 4 & $* *$ & & & & & \\
\hline $\begin{array}{l}\text { G. angustatum (Kützing) } \\
\text { Rabenhorst }\end{array}$ & - & - & - & $\mathrm{r}$ & - & $\mathrm{r}$ & - & $*$ & & $*$ & & & $*$ \\
\hline G. clavatum Ehrnberg & - & $\mathrm{r}$ & $\mathrm{r}$ & $\mathrm{r}$ & $\mathrm{r}$ & $\mathrm{r}$ & 3 & $* *$ & & & & & \\
\hline $\begin{array}{l}\text { G. olivaceum var. olivaceum } \\
\text { (Hornemann) Brébisson }\end{array}$ & $\mathrm{r}$ & $\mathrm{r}$ & $\mathrm{r}$ & $\mathrm{r}$ & $\mathrm{r}$ & $\mathrm{r}$ & 5 & $*$ & & & $*$ & & * \\
\hline G. parvulum (Kützing) Kützing & $\mathrm{r}$ & $\mathrm{r}$ & $\mathrm{r}$ & $\mathrm{u}$ & $\mathrm{u}$ & $\mathrm{r}$ & 3 & $*$ & $*$ & $*$ & $*$ & & $*$ \\
\hline G. truncatum Ehrenberg & - & - & - & $\mathrm{r}$ & $\mathrm{r}$ & - & 4 & $* *$ & & & & & \\
\hline $\begin{array}{l}\text { Gyrosigma attenuatum (Kützing) } \\
\text { Rabenhorst }\end{array}$ & $\mathrm{r}$ & $\mathrm{r}$ & $\mathrm{r}$ & - & $\mathrm{r}$ & - & 5 & $* *$ & & & & & \\
\hline G. peisonis (Grunow) Hustedt & $\mathrm{r}$ & - & $\mathrm{r}$ & $\mathrm{r}$ & $\mathrm{r}$ & $\mathrm{r}$ & 4 & $*$ & & $*$ & & & \\
\hline G. scalproides (Rabenhorst) Cleve & - & $\mathrm{r}$ & $\mathrm{r}$ & $\mathrm{r}$ & - & - & - & * & $*$ & & & & \\
\hline $\begin{array}{l}\text { Hantzschia virgata var. virgata } \\
\quad \text { (Roper) Grunow }\end{array}$ & - & - & - & - & $\mathrm{r}$ & $\mathrm{r}$ & - & $* *$ & & & & & \\
\hline
\end{tabular}




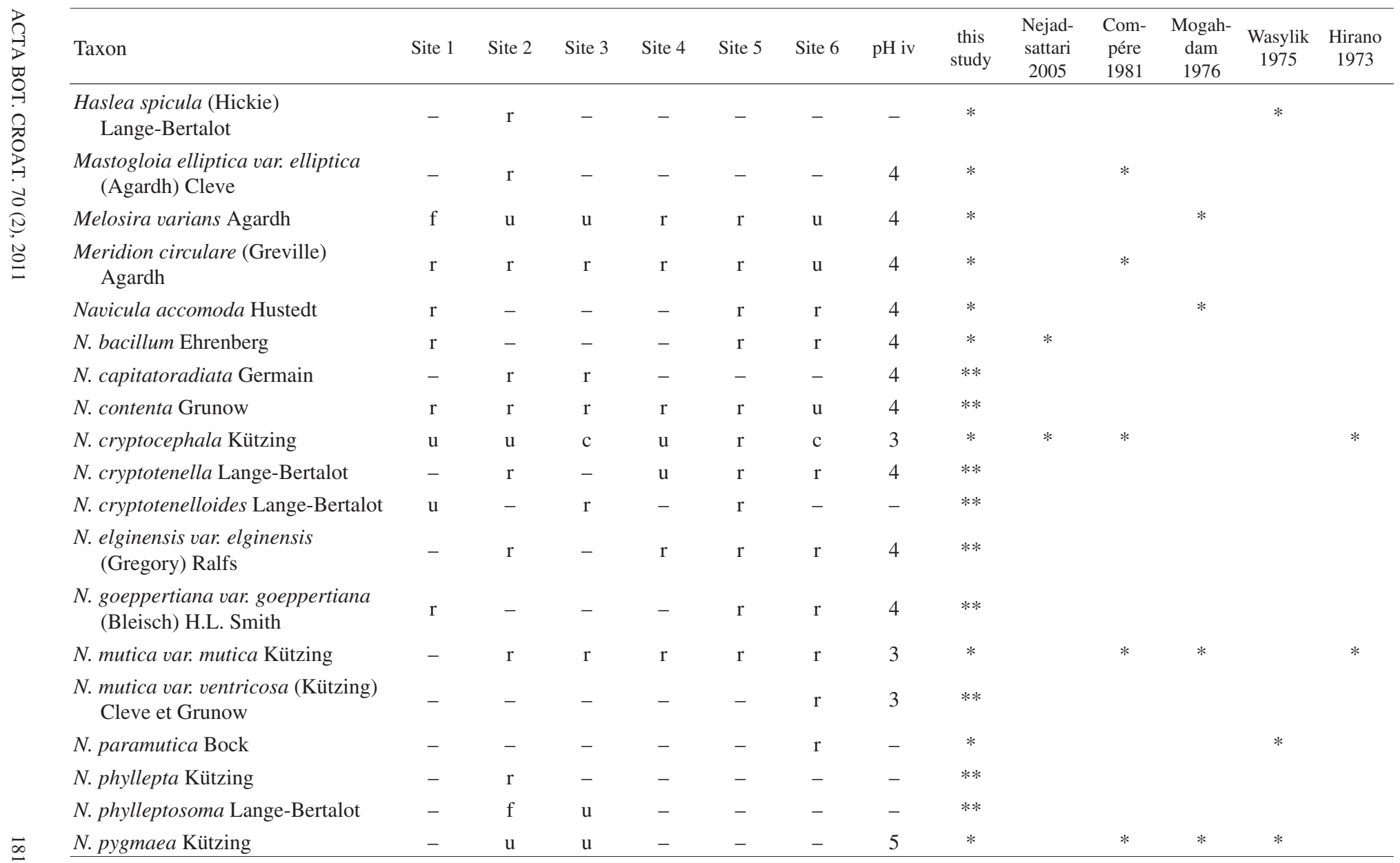




\begin{tabular}{|c|c|c|c|c|c|c|c|c|c|c|c|c|c|}
\hline Taxon & Site 1 & Site 2 & Site 3 & Site 4 & Site 5 & Site 6 & $\mathrm{pH}$ iv & $\begin{array}{l}\text { this } \\
\text { study }\end{array}$ & $\begin{array}{l}\text { Nejad- } \\
\text { sattari } \\
2005\end{array}$ & $\begin{array}{l}\text { Com- } \\
\text { pére } \\
1981\end{array}$ & $\begin{array}{c}\text { Mogah- } \\
\text { dam } \\
1976\end{array}$ & $\begin{array}{c}\text { Wasylik } \\
1975\end{array}$ & $\begin{array}{c}\text { Hirano } \\
1973\end{array}$ \\
\hline
\end{tabular}

$N$. radiosa Kützing

$N$. salinarum var. salinarum Grunow in Cleve et Grunow

N. subhamulata Hustedt

N. tripunctata (O.F. Müller) Bory

$N$. viridula var. viridula (Kützing) Ehrenberg

Nitzschia agnita Hustedt

N. amphibia f. amphibia Grunow

$N$. bicapitata Cleve

$N$. closterium (Ehrenberg) W. Smith

$N$. constricta (Kützing) Ralfs

$N$. dissipata var. dissipata (Kützing) Grunow

N. dissipata var. media (Hantzsch) Grunow

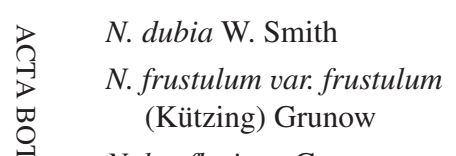

$N$. heufleriana Grunow

$N$. homburgiensis Lang-Bertalot

$N$. inconspicua Grunow

$N$. linearis var. tenuis (W. Smith) Grunow

\begin{tabular}{|c|c|c|c|c|c|c|c|c|c|c|c|}
\hline $\mathrm{r}$ & $\mathrm{r}$ & $\mathrm{r}$ & $\mathrm{r}$ & $r$ & $r$ & 3 & $*$ & T & & $*$ & \\
\hline $\mathrm{r}$ & $\mathrm{c}$ & $\mathrm{r}$ & - & - & $\mathrm{r}$ & 3 & $*$ & & & & $*$ \\
\hline- & $\mathrm{r}$ & - & - & - & - & 3 & $* *$ & & & & \\
\hline $\mathrm{c}$ & $\mathrm{r}$ & $\mathrm{u}$ & $\mathrm{u}$ & $\mathrm{r}$ & $\mathrm{u}$ & 4 & $*$ & & $*$ & $*$ & \\
\hline- & - & - & - & $\mathrm{r}$ & - & 4 & $*$ & $*$ & & & \\
\hline $\mathrm{r}$ & - & - & - & - & - & - & $* *$ & & & & \\
\hline- & - & - & $\mathrm{r}$ & - & - & 4 & $*$ & & $*$ & $*$ & \\
\hline - & - & - & - & - & $\mathrm{r}$ & - & $* *$ & & & & \\
\hline- & $\mathrm{r}$ & - & - & - & - & - & $*$ & & & $*$ & \\
\hline $\mathrm{r}$ & $\mathrm{u}$ & $\mathrm{f}$ & $\mathrm{r}$ & $\mathrm{r}$ & $\mathrm{r}$ & 4 & $* *$ & & & & \\
\hline $\mathrm{u}$ & $\mathrm{r}$ & $\mathrm{r}$ & $\mathrm{u}$ & $\mathrm{r}$ & $\mathrm{u}$ & 4 & $*$ & & $*$ & $*$ & \\
\hline- & - & - & - & - & $\mathrm{r}$ & 4 & $* *$ & & & & \\
\hline $\mathrm{r}$ & - & $\mathrm{r}$ & - & - & - & 3 & $*$ & & & $*$ & \\
\hline $\mathrm{r}$ & $\mathrm{r}$ & - & - & $\mathrm{r}$ & - & 4 & $*$ & & $*$ & $*$ & \\
\hline - & $\mathrm{r}$ & - & - & - & - & 4 & $* *$ & & & & \\
\hline- & - & - & - & - & $\mathrm{r}$ & 3 & $* *$ & & & & \\
\hline $\mathrm{r}$ & $\mathrm{u}$ & $\mathrm{c}$ & $\mathrm{r}$ & $\mathrm{r}$ & $\mathrm{r}$ & 4 & $* *$ & & & & \\
\hline$r$ & - & $\mathrm{c}$ & - & - & - & 3 & $* *$ & & & & \\
\hline $\mathrm{r}$ & $\mathrm{r}$ & $\mathrm{r}$ & - & $\mathrm{r}$ & $\mathrm{r}$ & - & $* *$ & & & & \\
\hline
\end{tabular}




\begin{tabular}{|c|c|c|c|c|c|c|c|c|c|c|c|c|c|}
\hline Taxon & Site 1 & Site 2 & Site 3 & Site 4 & Site 5 & Site 6 & $\mathrm{pH}$ iv & $\begin{array}{l}\text { this } \\
\text { study }\end{array}$ & $\begin{array}{l}\text { Nejad- } \\
\text { sattari } \\
2005\end{array}$ & $\begin{array}{c}\text { Com- } \\
\text { pére } \\
1981\end{array}$ & $\begin{array}{l}\text { Mogah- } \\
\text { dam } \\
1976 \\
\end{array}$ & $\begin{array}{l}\text { Wasylik } \\
1975\end{array}$ & $\begin{array}{c}\text { Hirano } \\
1973\end{array}$ \\
\hline N. palea (Kützing) W. Smith & $\mathrm{r}$ & - & $\mathrm{r}$ & $\mathrm{r}$ & $\mathrm{c}$ & $\mathrm{c}$ & 3 & $*$ & $*$ & $*$ & $*$ & & \\
\hline N. recta Hantzsch & $\mathrm{r}$ & $\mathrm{r}$ & $\mathrm{r}$ & $\mathrm{r}$ & $\mathrm{r}$ & $\mathrm{r}$ & 4 & $*$ & & & $*$ & & \\
\hline N. scalpelliformis Grunow & - & $\mathrm{r}$ & $\mathrm{r}$ & $\mathrm{r}$ & - & $\mathrm{r}$ & - & $*$ & & $*$ & & $*$ & \\
\hline N. sigma (Kützing) W. Smith & $\mathrm{r}$ & $\mathrm{r}$ & $\mathrm{r}$ & $\mathrm{r}$ & $\mathrm{r}$ & $\mathrm{r}$ & 4 & $*$ & & $*$ & $*$ & & \\
\hline $\begin{array}{l}\text { N. sinuata var. tabellaria (Grunow) } \\
\text { Grunow }\end{array}$ & - & - & - & $\mathrm{r}$ & $\mathrm{r}$ & - & 3 & $* *$ & & & & & \\
\hline $\begin{array}{l}\text { N. umbonata (Ehrenberg) } \\
\text { Lange-Bertalot }\end{array}$ & - & - & - & - & $\mathrm{r}$ & - & 3 & $* *$ & & & & & \\
\hline N. vermicularis (Kützing) Hantzsch & $\mathrm{r}$ & $\mathrm{r}$ & $\mathrm{r}$ & - & $\mathrm{r}$ & $\mathrm{r}$ & 4 & $*$ & * & & & & \\
\hline $\begin{array}{l}\text { Orthoseira roeseana (Rabenhorst) } \\
\text { O'Meara }\end{array}$ & - & $\mathrm{r}$ & $\mathrm{r}$ & - & $\mathrm{r}$ & $\mathrm{r}$ & - & $* *$ & & & & & \\
\hline $\begin{array}{l}\text { P. divergence var. divergence } \\
\text { W. Smith }\end{array}$ & - & - & - & - & $\mathrm{r}$ & - & 3 & $* *$ & & & & & \\
\hline $\begin{array}{l}\text { P. divergence var. elliptica (Grunow) } \\
\text { Cleve }\end{array}$ & - & - & $\mathrm{r}$ & - & - & - & - & $* *$ & & & & & \\
\hline P. lagerstedtii Cleve (Cleve-Euler) & - & - & - & - & - & $\mathrm{r}$ & 3 & $* *$ & & & & & \\
\hline $\begin{array}{l}\text { P. microstauron var. brebissonii } \\
\text { (Kützing) Mayer }\end{array}$ & $\mathrm{r}$ & - & $\mathrm{r}$ & - & - & - & 3 & $* *$ & & & & & \\
\hline P. obscura Krasske & - & - & - & - & - & $\mathrm{r}$ & 3 & $* *$ & & & & & \\
\hline P. schroederii (Hustedt) Krammer & $\mathrm{r}$ & - & - & - & - & - & - & $* *$ & & & & & \\
\hline P. viridis (Nitzsch) Ehrenberg & - & - & $\mathrm{r}$ & $\mathrm{r}$ & - & $\mathrm{r}$ & 3 & $*$ & & $*$ & & & \\
\hline
\end{tabular}




\begin{tabular}{|c|c|c|c|c|c|c|c|c|c|c|c|c|c|}
\hline Taxon & Site 1 & Site 2 & Site 3 & Site 4 & Site 5 & Site 6 & $\mathrm{pH}$ iv & $\begin{array}{l}\text { this } \\
\text { study }\end{array}$ & $\begin{array}{c}\text { Nejad- } \\
\text { sattari } \\
2005\end{array}$ & $\begin{array}{c}\text { Com- } \\
\text { pére } \\
1981\end{array}$ & $\begin{array}{c}\text { Mogah- } \\
\text { dam } \\
1976\end{array}$ & $\begin{array}{c}\text { Wasylik } \\
1975\end{array}$ & $\begin{array}{c}\text { Hirano } \\
1973\end{array}$ \\
\hline
\end{tabular}

Pleurosigma salinarum Grunow

Pleurosira laevis f. polymorpha (Kützing) Compére

Rhoicosphenia abbreviata (Agardh)

Lange-Bertalot

Rhopalodia acuminata Krammer

R. gibba var. gibba (Ehrenberg) O. Müller

R. rupestris (W. Smith) Krammer

Stauroneis acuta var. acuta

W. Smith

S. anceps var. anceps Ehrenberg

Stenopterobia curvula (W. Smith) Krammer

Surirella amphioxys W. Smith

S. angusta Kützing

S. brebissonii var. brebissonii Krammer et Lange-Bertalot

S. brebissonii var. kuetzingii Krammer et Lange-Bertalot

S. brebissonii var. punctata Krammer

S. minuta Brébisson

S. ovalis Brébisson

Synedra gaillonii (Bory) Ehrenberg

\begin{tabular}{|c|c|c|c|c|c|c|c|c|c|c|c|c|}
\hline $\mathrm{r}$ & $\mathrm{r}$ & - & - & - & - & 4 & $*$ & & * & & & \\
\hline $\mathrm{r}$ & $\mathrm{r}$ & - & - & - & - & 5 & $* *$ & & & & & \\
\hline c & $\mathrm{r}$ & $\mathrm{r}$ & $\mathrm{r}$ & $\mathrm{r}$ & $\mathrm{u}$ & 4 & $*$ & $*$ & $*$ & $*$ & & \\
\hline $\mathrm{r}$ & $\mathrm{r}$ & - & - & - & - & - & $* *$ & & & & & \\
\hline- & - & - & $\mathrm{r}$ & - & - & 5 & $*$ & & $*$ & & & \\
\hline- & $\mathrm{r}$ & $\mathrm{r}$ & - & - & - & - & $* *$ & & & & & \\
\hline- & - & $\mathrm{r}$ & - & - & $\mathrm{r}$ & 4 & $* *$ & & & & & \\
\hline $\mathrm{r}$ & $\mathrm{r}$ & - & - & - & $\mathrm{r}$ & 3 & $*$ & $*$ & $*$ & & $*$ & \\
\hline - & $\mathrm{r}$ & $\mathrm{r}$ & - & - & - & 2 & $* *$ & & & & & \\
\hline - & - & $\mathrm{r}$ & $\mathrm{r}$ & - & - & 4 & $* *$ & & & & & \\
\hline $\mathrm{r}$ & $\mathrm{r}$ & $\mathrm{r}$ & $\mathrm{r}$ & $\mathrm{r}$ & $\mathrm{r}$ & 4 & $*$ & & $*$ & $*$ & & \\
\hline $\mathrm{r}$ & $\mathrm{r}$ & $\mathrm{r}$ & - & $\mathrm{r}$ & - & 4 & $* *$ & & & & & \\
\hline - & - & $\mathrm{r}$ & - & - & - & 4 & $*$ & & & $*$ & & \\
\hline $\mathrm{r}$ & - & - & - & - & - & - & $* *$ & & & & & \\
\hline $\mathrm{r}$ & $\mathrm{r}$ & $\mathrm{r}$ & $\mathrm{r}$ & $\mathrm{r}$ & $\mathrm{r}$ & 4 & $* *$ & & & & & \\
\hline $\mathrm{u}$ & $\mathrm{r}$ & $\mathrm{c}$ & $\mathrm{r}$ & $\mathrm{r}$ & $\mathrm{u}$ & 4 & $*$ & & $*$ & $*$ & & $*$ \\
\hline - & - & $\mathrm{r}$ & $\mathrm{r}$ & - & - & - & $* *$ & & & & & \\
\hline
\end{tabular}


Table 3. Relative abundance of diatom species present in this study calculated and summed up for the six sites in Ramsar (300 valves were calculated at each site).

\begin{tabular}{lclc}
\hline $\begin{array}{l}\text { Most abundant } \\
\text { genera }\end{array}$ & $\begin{array}{c}\text { Median (min, max) } \\
\text { abundance }\end{array}$ & \multicolumn{1}{c}{ Most abundant species } & $\begin{array}{c}\text { Median (min, max) } \\
\text { abundance }\end{array}$ \\
\hline Achnanthes & $17(0.2-52)$ & Achnanthes minutissima var. Jackii & $16(0.0-51)$ \\
Nitzschia & $11(3.6-64)$ & Navicula phylleptosoma & $0.0(0.0-40)$ \\
Navicula & $9.6(1.1-65)$ & Melosira varians & $1.9(0.4-32)$ \\
Cocconeis & $9.1(0.3-28)$ & Cocconeis placentula var. euglypta & $5.2(0.2-12)$ \\
Melosira & $1.9(0.4-32)$ & Nitzschia palea & $0.6(0.0-17)$ \\
Amphora & $5.1(0.6-18)$ & Nitzschia constricta & $0.0(0.0-27)$ \\
Craticula & $0.0(0.0-19)$ & Cocconeis pediculus & $0.8(0.0-16)$ \\
Diatoma & $0.9(0.0-1.9)$ & Amphora pediculus & $1.7(0.4-11)$ \\
Rhoicosphenia & $0.9(0.2-11)$ & Navicula salinarum var. salinarum & $0.3(0.0-20)$ \\
Surirella & $0.9(0.1-11)$ & Craticula buderi & $0.0(0.0-19)$ \\
Cymbella & $1.1(0.0-10)$ & Nitzschia inconspicua & $0.7(0.2-17)$ \\
Diploneis & $0.2(0.0-5.4)$ & Navicula cryptocephala & $2.5(0.7-6.2)$ \\
\hline
\end{tabular}

which was followed by site 6 ( 83 taxa). The lowest diversities were found at sites 4 and 1 (66 and 73 taxa respectively). Thirty species were present at all sites, and among them Nitzschia and Navicula were the most diverse genera (five and four taxa respectively). Achnanthes and Surirella (three taxa) and Amphora, Cocconeis, Cymbella, Diploneis, Fragilaria and Gomphonema (two taxa) and Melosira, Meridion and Rhoicosphenia were present with only one taxon each (Tab. 2).

\section{Distribution and occurrence of species}

Of the 153 identified taxa in this study, seventeen taxa, Amphora ovalis, Caloneis bacillum, Cocconeis placentula var. euglypta, Cyclotella meneghiniana, Cymatopleura elliptica var. elliptica, Cymatopleura solea var. solea, Cymbella helvetica, Fragilaria ulna var. ulna, Gomphonema angustatum, Gomphonema olivaceum var. olivaceum, Gomphonema parvulum, Navicula cryptocephala, Navicula mutica var. mutica, Navicula salinarum var. salinarum, and Nitzschia sigmoidea were recorded at nine sampling sites in Iran by Hirano (1973). Seven diatom species Amphora commutata, Cymbella affinis, Haslea spicula, Navicula paramutica, Navicula pygmaea, Nitzschia scalpelliformis and Stauroneis anceps var. anceps were reported by WASYLIK (1975) from different areas of Iran.

Moghadam (1976) observed a total of 38 species from the Zayandeh Rud in central Iran. Certain of these taxa were also observed in the present study (Amphora ovalis, Bacillaria paradoxa, Cocconeis placentula var. euglypta, Cyclotella meneghiniana, Diatoma vulgaris morphotype vulgaris, Fragilaria ulna var. acus, Fragilaria ulna var. ulna, Gomphonema olivaceum var. olivaceum, Gomphonema parvulum, Melosira varians, Navicula pygmaea, Nitzschia frustulum var. frustulum, Nitzschia palea, Nitzschia sigmoidea, Surirella angusta). 
COMPÈRE (1981) reported 43 taxa from 21 localities in the central and southeastern parts of Iran. The following taxa were also found in the present study: Amphora ovalis, Cocconeis pediculus, Cyclotella meneghiniana, Cymbella helvetica, Diatoma vulgaris morphotype vulgaris, Fragilaria ulna var. acus, Fragilaria ulna var. ulna, Gomphonema angustatum, Gomphonema parvulum, Navicula pygmaea, Nitzschia frustulum var. frustulum, Nitzschia palea, Nitzschia sigmoidea, Stauroneis anceps var. anceps and Surirella angusta.

Among diatom species presented in this study, 16 taxa (Amphora ovalis, Cyclotella meneghiniana, Cymatopleura elliptica var. elliptica, Diatoma vulgaris morphotype vulgaris, Fragilaria ulna var. acus, Fragilaria ulna var. ulna, Gomphonema parvulum, Gyrosigma scalproides, Navicula bacillum, Navicula cryptocephala Navicula viridula var. viridula, Nitzschia palea, Nitzschia sigmoidea, Nitzschia vermicularis, Rhoicosphenia abbreviate, Stauroneis anceps var. anceps) were previously reported by NEJADSATTARI (2005) from Lake Neure in the Ardebil province of Eastern Azarbijan, Iran.

\section{Ecology of the more commonly occurring taxa}

The following ecological requirements are based on VAN DAM et al. (1994).

\section{Achnanthes minutissima var. jackii (Rabenhorst) Lange-Bertalot 1980}

Distribution: This taxon was the most abundant species at site 4 with $38.3 \%$, site 5 with more than $50 \%$ and site 6 with $32 \%$ of population (Tab. 1).

Ecology: Circumneutral mainly occurring at $\mathrm{pH}$ values about 7, fresh -brackish, sometimes in oligosaprobe waters, with mid- to weak light electrolytic conditions.

\section{Amphora pediculus (Kützing) Grunow 1880}

Distribution: Highest percentage (11\%) occurred at site 6, and was present with low numbers at other sites.

Ecology: Alcaliphilic, mainly occurring at $\mathrm{pH}>7$, fresh - brackish, oxygen, fairly high (above $75 \%$ saturation), $\beta$-mesosaprobous, nitrogen - autotrophic taxon, tolerating elevated concentrations of organically bound nitrogen. Mainly occurring in water bodies, also rather regularly on wet and moist places.

\section{Cocconeis pediculus Ehrenberg 1838}

Distribution: Constitutes $10.4 \%$ of diatom population at site 1, but was second most abundant with $16.3 \%$ at site 4 , occurring at other sites with low numbers.

Ecology: Alcaliphilic, mainly occurring at $\mathrm{pH}>7$, fresh - brackish, oxygen, moderate (above 50\% saturation), $\beta$-mesosaprobous, nitrogen - autotrophic taxon, tolerating concentrations of organically bound nitrogen, hyper-eutraphentic. Never, or only very rarely, occurring outside water bodies.

\section{Cocconeis placentula var. euglypta (Ehrenberg 1854) Grunow 1884}

Distribution: Occurring at all sites, highest numbers were found at sites 4 and 6, with $12 \%$ and $11 \%$ respectively. 
Ecology: Alcaliphilic, mainly occurring at $\mathrm{pH}>7$, fresh - brackish, salt intolerant to indifferent. Oxygen, moderate (above 50\% saturation), $\beta$-mesosaprobous, nitrogen - autotrophic taxon, tolerating concentrations of organically bound nitrogen, hyper-eutraphentic. Occurring mainly in water bodies and sometimes on wet places.

\section{Craticula buderi (Hustedt) Lange-Bertalot 2000}

Distribution: With $19.3 \%$ of the population at site 5, this was the second dominant species, but was absent at other sites with the exception of site 1 with $1 \%$.

Ecology: It is common in freshwaters with average to higher electrolyte content, e.g. chalk rich springs. Its tolerance to trophy and saprobity is very broad, ranging from weakly meso - to hypereutrophic, and from oligo- to $\alpha$-mesosaprobic. It is also found in periodically wet habitats, e.g. moist meadows and in industrial effluent.

\section{Melosira varians Agardh 1827}

Distribution: Dominant species with $32.3 \%$ at site 1 , occurring with small numbers at other sites.

Ecology: Alcaliphilic, mainly occurring at $\mathrm{pH}>7$, optimum about 8.5. Fresh - brackish, oxygen, moderate (above 50\% saturation), $\alpha$-meso/ polysaprobous, facultatively nitrogen - heterotrophic taxon, needing periodically elevated concentrations of organically bound nitrogen, eutraphentic. Mainly occurring in water bodies, also rather regularly on wet and moist places.

\section{Navicula cryptocephala Kützing 1844}

Distribution: Occurring in low numbers at all sampling sites with highest numbers at site $6(7 \%)$ and lowest at site 5 (less than $1 \%)$.

Ecology: Circumneutral mainly occurring at $\mathrm{pH}$ values about 7 , fresh-brackish, oxygen, moderate (above 50\% saturation), $\beta$-mesosaprobous, nitrogen - autotrophic taxon, tolerating elevated concentrations of organically bound nitrogen, oligo- to meso- to rarely and weakly eutraphentic, Mainly occurring in water bodies, sometimes on wet places.

Navicula phylleptosoma Lange-Bertalot in Lange-Bertalot et Genkal 1999

Distribution: Dominant species with $40 \%$ of total population at site 2 , but was absent at other sites with the exception of site $3(1 \%)$.

Ecology: Occurs at $\mathrm{pH}$ values of about 7, fresh-brackish, presumably cosmopolitan. Occurrence is not precisely known, scattered throughout Europe (LANGE-BERTALOT 2001); occurs in electrolyte rich freshwaters.

Navicula salinarum var. salinarum Grunow in Cleve et Grunow 1880

Distribution: This taxon was the second most abundant at site 2, but almost absent at other sites.

Ecology: Circumneutral mainly occurring at $\mathrm{pH}$ values about 7 , found in fresh and brackish waters, prefers water of high mineral content, oxygen, fairly high (above $75 \%$ sat- 
uration), $\beta$-mesosaprobous, nitrogen - autotrophic taxon, tolerating elevated concentrations of organically bound nitrogen, eutraphentic. Never, or only very rarely, occurring outside water bodies.

\section{Nitzschia constricta (Kützing) Ralf in Pritchard 1861}

Distribution: Dominant taxon at site 3 with $27.3 \%$, but almost absent at other sites with the exception of site 2 which consist $2 \%$ of population.

Ecology: Alcaliphilic, mainly occurring at $\mathrm{pH}>7$, cosmopolitan, found in brackish and freshwaters, oxygen, moderate (above 50\% saturation), $\alpha$-mesosaprobous, nitrogen autotrophic taxon, tolerating elevated concentrations of organically bound nitrogen, hyper-eutraphentic. Occurring mainly in water bodies, sometimes on wet places.

\section{Nitzschia inconspicua Grunow 1862}

Distribution: Highest number was found at site 3 with $16.3 \%$, but occurred in very low numbers (less than $1 \%$ ) at other sites.

Ecology: Alcaliphilic, mainly occurring at $\mathrm{pH}>7$, cosmopolitan, found in brackish and freshwaters, oxygen, moderate (above $50 \%$ saturation), $\alpha-\beta$-mesosaprobous, nitrogen autotrophic taxon, tolerating elevated concentrations of organically bound nitrogen. Oligo - to eutraphentic (hyper-eutraphentic). In waters of mid- to high electrolytic conditions, can withstand osmotic fluctuations typical of sapro-littoral zones, tolerant of high organic loading. Mainly occurring in water bodies, also rather regularly on wet and moist places.

\section{Nitzschia palea (Kützing) W.Smith 1856}

Distribution: Occurring with $16.6 \%$ was second most dominant taxon at site 6 and with relatively high numbers at site 5 with $14 \%$, but very low numbers at other sites (Tab. 1).

Ecology: Circumneutral mainly occurring at $\mathrm{pH}$ values about 7 , fresh-brackish, oxygen, low (above 30\% saturation), polysaprobous. Obligately nitrogen heterotrophic taxa, constantly needing elevated concentrations of organically bound nitrogen, hyper-eutraphentic. Mainly occurring in water bodies, also rather regularly on wet and moist places. $N$. palea typically has its greatest development in polluted waters. Tolerant of high level of organic waste and is sometimes found in association with industrial waste. Tolerant of a wide range of trophic and electrolytic conditions.

The epilithic diatoms of the Ramsar streams were diverse and dominated by taxa found in meso - to fairly eutrophic waters. The species abundance observed in this study was high. The result from this study suggests that as more studies are performed in the region along the coast of Caspian Sea, many more diatom taxa will be added to the list of Persian diatoms. It should be noted that the current study was based on a single day's sampling in a single year. This limited information does not allow any in-depth conclusion on the epilithic diatom community in Ramsar. In order to obtain a better estimation of diatom diversity, further studies need to be carried out in the region and promise to greatly increase this diversity. 


\section{Acknowledgements}

We thank Mr E. Tulisalo, M.Sc. and Ms L. Montonen, M.Sc. at the University of Helsinki and the staff at Alfred Wegener Institute for valuable assistance in sample preparation, microscoping and identification. Our special thanks to Dr. A. Maassomi for guidance and help in gathering sample materials. The help and assistance of Ms T. Kettunen processing the pictures using Photoshop is acknowledged as well as valuable comments and support from Mr. Tinat M. A.

\section{References}

Alfinito, S., Fumanti, B., 1980: Contribution to the knowledge of freshwater algae from lake Hamun-i-puzak (Iran). Nova Hedwigia 33, 873-882.

Atazadeh, I., Sharifi, M., Kelly, M. G., 2007: Evaluation of the trophic diatom index for assessing water quality in River Gharasou, western Iran. Hydrobiologia 589, 165-173.

BozorgniA, H. 1970: Geological map of south falank of east Alborz 1:100000. National, Iran Oil Co. Geology Report, Tehran.

Compère, P., 1981: Algues des déserts d'Iran. Bulletin de Jardin Botanique Nationale de Belgique 51, 3-40.

Fore, L. S., Grafe, C., 2002: Using Diatoms to assess the biological conditions of large rivers in Idaho (USA). Freshwater Biology 47, 2015-2037.

Hafezi Moghaddas, N., Gafoori, M., 2007: Investigation of the distribution and causes of landslides in Central Alborz, Iran. World Applied Sciences Journal 2, 652-657.

HirAnO, M., 1973: Freshwater algae from Mesopotamia. Contributions of Biological Laboratory of Kyoto University 24, 105-119.

Kimball, K. D., Kimball, S. F., 1977: Seasonal phytoplankton variation in the shallow Pahlavi Mordab, Iran. Hydrobiologia 55, 49-53.

Khan, A. A., Ali, S. B., 2003: Effects of erosion on Indus River bio-diversity in Pakistan. Pakistan Journal of Biological Sciences 6, 1035-1040.

Krammer, K., 1992: Pinnularia. Eine Monographie der Europäischen Taxa. Bibliotheca Diatomologica 9, 1-353.

Krammer, K., 1997a: Die cymbelloiden Diatomeen. Eine Monographie der weltweit bekannten Taxa, 1. Allgemeines und Encyonema Part. Bibliotheca Diatomologica 36, $1-382$.

KrAmMER, K., 1997b: Die cymbelloiden Diatomeen. Eine Monographie der weltweit bekannten Taxa, 2. Encyonema Part, Eucyonopsis and Cymbellopsis. Bibliotheca Diatomologica 37, 1-469.

KrAmmer, K., 2002: Cymbella. Diatoms of Europe. Diatoms of the European inland waters and comparable habitats 3, 1-584.

Krammer, K., Lange-Bertalot, H., 1986: Bacillariophyceae, 1. Naviculaceae. In: EtTL, H., Gerloff, J., Heynig, H., Mollenhauer, D. (eds), Süsswasserflora von Mitteleuropa, 2, 1, 1-876. Gustav Fischer Verlag. Jena. 
Krammer, K., Lange-Bertalot, H., 1988: Bacillariophyceae, 2. Bacillariaceae, Epithemiaceae, Surirellaceae. In: Ettr, H., Gerloff, J., Heynig, H., Mollenhauer, D. (eds), Süsswasserflora von Mitteleuropa, 2, 2, 1-596. Gustav Fischer Verlag. Stuttgart.

Krammer, K., Lange-Bertalot, H. 1991a: Bacillariophyceae, 3. Centrales Fragilariaceae, Eunotiaceae. In: Ettl, H., Gerloff, J., Heynig, H., Mollenhauer, D. (eds), Süsswasserflora von Mitteleuropa, 2, 3, 1-576. Gustav Fischer Verlag. Stuttgart.

Krammer, K., LANGE-Bertalot, H., 1991b: Bacillariophyceae, 4. Achnanthaceae. Kritische Ergänzungen zu Navicula (Lineolatae) und Gomphonema. In: ETTL, H., GERLOFF, J., Heynig, H., Mollenhauer, D. (eds), Süsswasserflora von Mitteleuropa, 2, 4, 1-437. Gustav Fischer Verlag. Stuttgart.

LAnge-Bertalot, H., 1993: 85 neue Taxa und über 100 weitere neu definierte Taxa ergänzend zur Süsswasserflora von Mitteleuropa, 2, 1-4. Bibliotheca Diatomologica $27,1-454$.

LANGE-BerTAlot, H., 2001: Navicula sensu lato. Frustulia. Diatoms of Europe. Diatoms of European Inland Waters and Comparable Habitats 2, 1-526.

LANGe-Bertalot, H., Metzelin, D., 1996: Oligotrophie-Indikatoren, 800 Taxa repräsentativ für drei diverse Seen-Typen, kalkreich - oligodystroph - schwach gepuffertes Weichwasser. Iconographia Diatomologia 2, 1-390.

Moghadam, F., 1976: Diatoms as indicator of pollution Zayandeh River, Iran. Proceedings of the Academy of Natural Sciences of Philadelphia 127, 281-297.

Nejadsattari, T., 2005: The diatom flora of Lake Neure, Iran. Diatom Research 20, 313-333.

Nejadsattari, T., Noroozi, M., Fallahi, M., 2005: The composition and seasonal distribution of epiphytic algae in Anzali lagoon, Iran. Cryptogamie Algologie 26, 387-398.

PATRICK, R., ReIMER, C. W., 1966: The diatom of the United State, exclusive of Alaska and Hawaii, 1. Fragilariaceae, Eunotiaceae, Achnanthaceae, Naviculaceae. Academy of Natural Sciences of Philadelphia, Monograph 13, 1-688.

REICHARDT, E., 1999: Zur Revision der Gattung Gomphonema. Iconographia Diatomologica $8,1-203$.

SoltanPour-Gargari, A., 1973: A survey on the limnology of Valasht Lake (Iran) and its phytoplankton population (in Persian). MScThesis, Tehran University. Tehran.

Van Dam, H., Mertens, A., Sinkeldam, J., 1994: A coded checklist and ecological indicator values of freshwater diatoms from the Netherlands. Netherlands Journal of Aquatic Ecology 28, 117-133.

VeselÁ, J., JohAnsen, J. R., 2009: The diatom flora of ephemeral headwater streams in the Elbsandsteingebirge region of the Czech Republic. Diatom Research 24, 443-477.

WASYLIK, K., 1975: Notes on the freshwater algae of Iran. Fragmenta Floristica et Geobotanica 21, 369-396.

Wasylikowa, A., WitKowski, A., 2008: The palaeoecology of Lake Zaribar and surrounding areas western Iran, during the 48000 years. Diatom Monographs 8, 1-378. 